\title{
Field Treatments for Actinic Keratosis: A Systematic Review and Network Meta-Analysis
}

Authors: Martin $\mathrm{G}^{1}$, Berman $\mathrm{B}^{2}$, Feldman $\mathrm{S}^{3}$, Armstrong A4, Edwards $\mathrm{M}^{5}$, Graziadio $\mathrm{S}^{5}$, McCool R $\mathrm{R}^{5}$, Arber $\mathrm{M}^{5}$, Carr $\mathrm{E}^{5}$, James $\mathrm{D}^{6}$, Chapman-Rounds $\mathrm{M}^{6}$, Fumero $\mathrm{E}^{7}$, Schuchardt $\mathrm{M}^{7}$, Grada $\mathrm{A}^{8}$ (*corresponding author: ayman.grada@almirall.com)

Dr. George Martin Dermatology Associates

University of Miami Miller School of Medicine, Miami, Florida and Center for Clinical and Cosmetic Research, Aventura Florida

3 Professor of Dermatology, Pathology, and Social Sciences \& Health Policy Wake Forest University Health Sciences, Winston-Salem, NC, USA

Southern California Clinical and Translational Science Institute, and Keck School of Medicine at University of Southern California, USA

\section{BACKGROUND AND OBJECTIVES}

Actinic keratosis (AK) is a pre-malignant skin lesion caused by long-term sun exposure. If untreated, AK may progress to squamous cell carcinoma, so early and effective treatment is essential. There is a lack of direct comparative studies for existing field therapies for AK, and our systematic review (SR) and network meta-analysis (NMA) aimed to generate indirect comparative data for the available treatments.

We conducted a SR and NMA to compare the evidence from randomized controlled trials (RCTs) on the efficacy, safety, and discontinuations of key field-directed treatments for AK (5-FU, imiquimod, ingenol mebutate, diclofenac sodium, PDT), plus a novel topical treatment, tirbanibulin.

\section{METHODS}

Eligible interventions were topical therapies, photodynamic therapy (PDT) following the use of sensitizer cream, and cryotherapy. Eight databases, two trials registers, five websites and a selection of conference proceedings were searched between June and September 2020. Following a feasibility assessment, a Bayesian network meta-analysis (NMA) was conducted, reporting odds ratios and credible intervals (i.e. confidence intervals in the Bayesian framework) for complete clearance and safety outcomes.

Two sets of analyses were proposed; primary analyses would include only the results of studies offering participants one course or session of treatment (a trial design comparable with that of the two tirbanibulin RCTs), while the secondary analyses would include results of studies offering any number of courses or sessions of treatment, plus retreatment where offered.

\section{Figure 1: Network Diagrams: Complete Clearance}

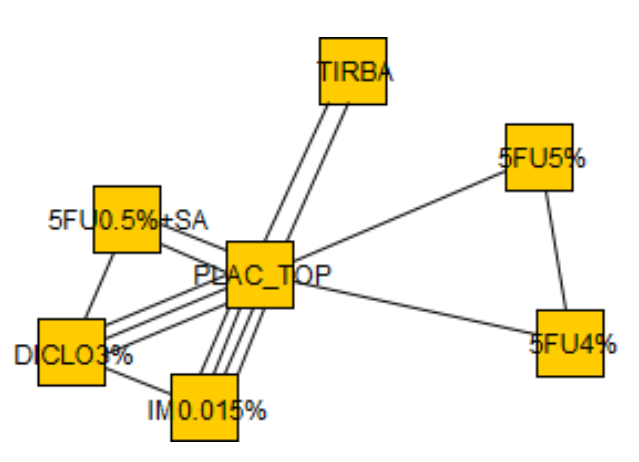

a. Primary analysis

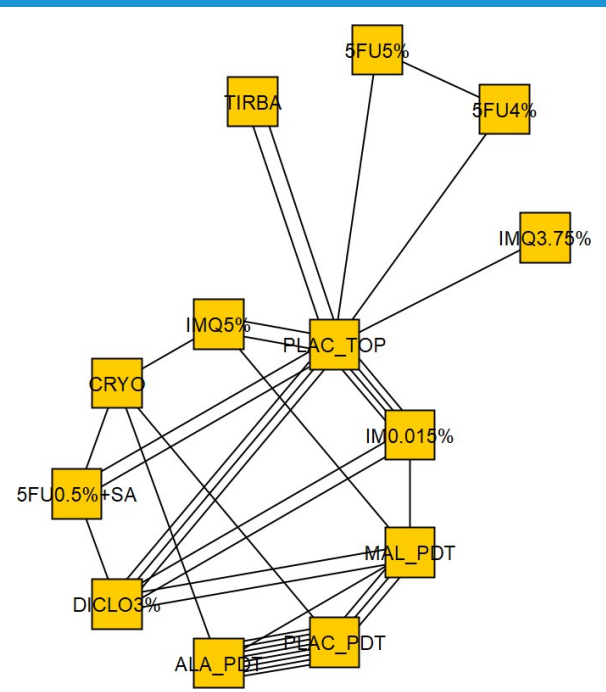

b. Secondary analysis RESULTS

Searches identified 4,128 records. Following assessment, 83 documents, reporting on 46 studies, were included. Six of these studies were not suitable for inclusion in the NMA due to insufficient or inappropriate reporting of data, or a lack of similarity with the other studies.

Feasibility assessment confirmed that the level of similarity between the studies to be included in the NMA was acceptable. Between-study heterogeneity was generally low $(<40 \%)$, but for a comparison between tirbanibulin and topical placebo, it was moderate $(56 \%)$. Sensitivity analysis did not raise major concerns; random effects models and weakly informative prior distributions were used to allow for this heterogeneity. The third NMA assumption is consistency between direct and indirect comparisons, and its assessment did not raise any major concerns.

Complete clearance was the only suitable outcome for NMA, with the primary analysis conducted on studies assessing one course or session of treatment. In addition to topical placebo, six interventions were included in the network (see Figure 1a)

Providing Consultancy \&

Research in Health Economics
5 York Health Economics Consortium, York, UK

6 Quantics Biostatistics, Edinburgh, UK

Almirall, Barcelona, Spain

Almirall (US)

\section{RESULTS (continued)}

A secondary analysis of studies (assessing any number of courses or sessions of treatment; see Figure $1 \mathrm{~b}$ ) enabled the addition to the analysis of one placebo and five active treatments.

The primary analysis showed that diclofenac $3 \%$ was the least efficacious of the active treatments (Figure 2). Based on the overlap of credible intervals shown in the statistical plots, the novel topical treatment (tirbanibulin $1 \%$ ointment) appeared to be as efficacious as existing treatments, and more so than topical placebo or diclofenac $3 \%$. Results of the secondary analysis (Figure 3) confirmed these findings, with diclofenac and placebo PDT appearing substantially less efficacious than the remaining non-placebo treatments.

\section{Figure 2: $\quad$ Results of the Primary Analysis}

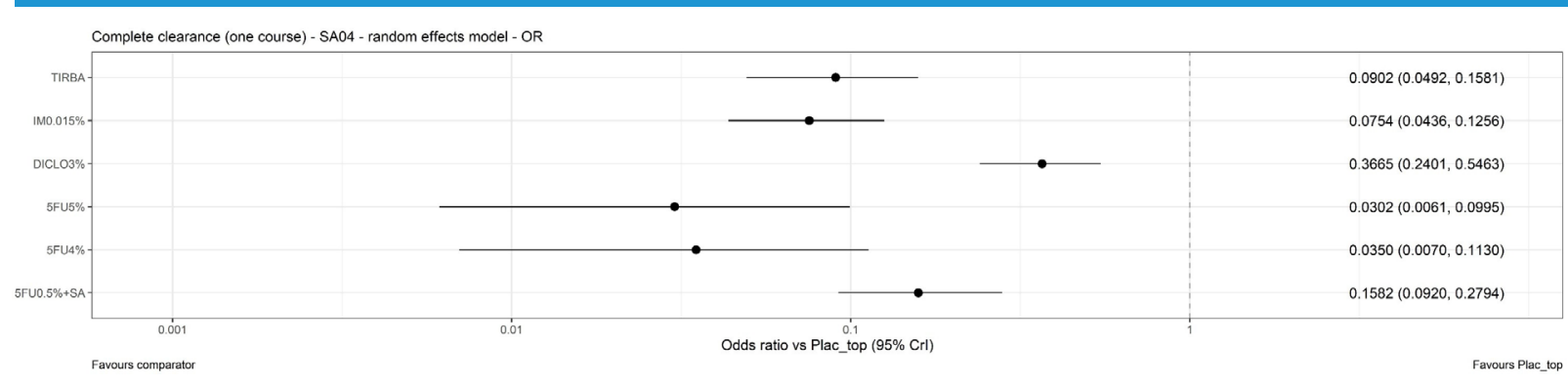

\section{Figure 3: Results of the Secondary Analysis}

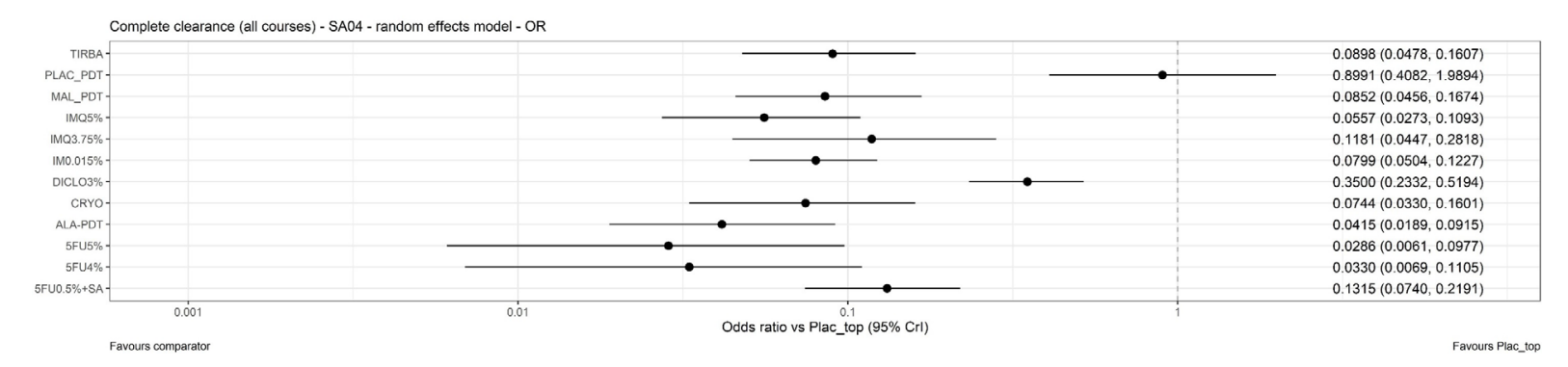

Although the review captured data on the incidence of severe local skin reactions (LSRs), the number of studies reporting each of the individual severe LSRs was relatively low, with a maximum of five studies reporting an individual severe LSR for the primary analysis. This data was not sufficient to support NMA of the incidence of any severe LSRs.

A qualitative assessment of discontinuations due to treatment-related adverse events (TRAEs) showed that in the primary analysis rates were between $1.4 \%$ (PDT with 5aminolevulinic acid sensitizer) and $8.2 \%$ (imiquimod $5 \%$ for 16 weeks) in all active arms. Tirbanibulin showed no discontinuations due to TRAEs out of 353 patients across two RCTs, and each of the severe LSRs were observed in $<10 \%$ of patients, with no skin complications (hypo/hyper-pigmentation, scarring) observed. Due to the zero counts it was not possible to conduct NMA of the outcome, but the qualitative assessment indicates that the safety profile of the new treatment is favourable.

\section{CONCLUSIONS}

Given the overlap between credible intervals shown in the statistical plots, treatment efficacy appeared similar for all active treatments. All treatments, including tirbanibulin, were better than placebo and diclofenac $3 \%$. Based on the zero rate of discontinuations due to TRAEs and the low percentage of severe LSRs, tirbanibulin appears to have a particularly favourable safety profile.

\section{CONTACT US}

Email: yhec@york.ac.uk

Website: www.yhec.co.uk

F http://tinyurl.com/yhec-facebook

3 http://twitter.com/YHEC1

GMINERVA http://www.minerva-network.com/ in http://tinyurl.com/YHEC-Linkedln

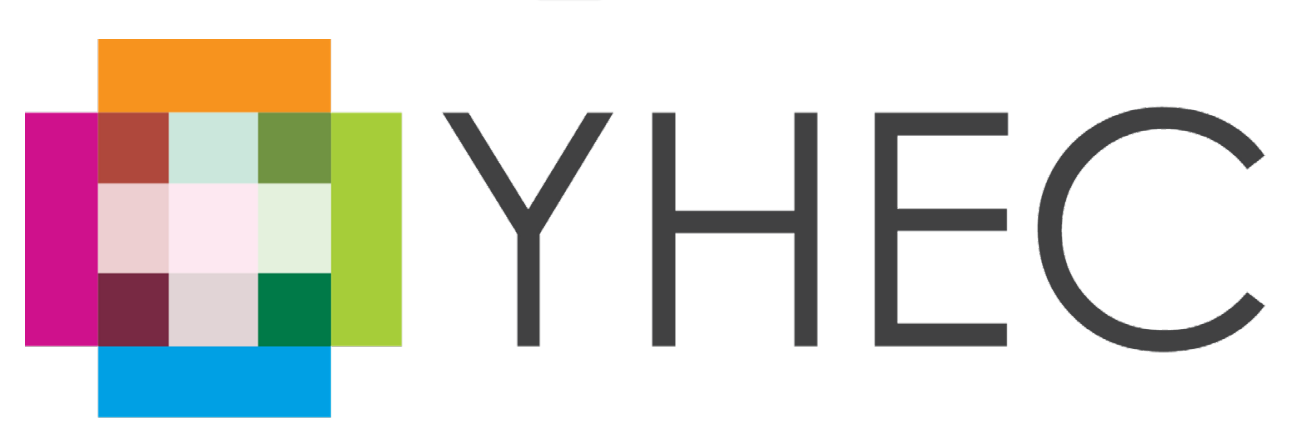

